OPEN ACCESS

Edited by:

Xiaoming Jin,

Indiana University-Purdue University Indianapolis, United States

Reviewed by:

Emmanuel N. Pothos,

Tufts University School of Medicine,

United States

Derya Dikmen,

Hacettepe University, Turkey

*Correspondence:

Brigitte Leeners

brigitte.leeners@usz.ch

Larissa S. Heuberger

larissa_heuberger@hotmail.com

${ }^{t}$ These authors share first authorship

Specialty section:

This article was submitted to

Gut-Brain Axis,

a section of the journal

Frontiers in Neuroscience

Received: 24 October 2021

Accepted: 21 January 2022

Published: 16 February 2022

Citation:

Heuberger LS, Gobbi S, Weber SC, Graf G, Tobler PN,

Asarian L, Geary N, Roth M and Leeners $B$ (2022) Is It Worth it? Obesity Affects Snack Food Valuation

Across the Menstrual Cycle.

Front. Neurosci. 16:800976.

doi: 10.3389/fnins.2022.800976

\title{
Is It Worth It? Obesity Affects Snack Food Valuation Across the Menstrual Cycle
}

\begin{abstract}
Larissa S. Heuberger ${ }^{1 *}$, Susanna Gobbi ${ }^{2+}$, Susanna C. Weber ${ }^{2}$, Gwendolyn Graf ${ }^{3}$, Philippe N. Tobler ${ }^{1,2}$, Lori Asarian ${ }^{4}$, Nori Geary ${ }^{5}$, Mareike Roth ${ }^{3}$ and Brigitte Leeners ${ }^{3,6 *}$

${ }^{1}$ Swiss Federal Institute of Technology, Zurich, Switzerland, ${ }^{2}$ Zurich Center for Neuroeconomics, University of Zurich, Zurich, Switzerland, ${ }^{3}$ Department of Reproductive Endocrinology, University Hospital of Zurich, Zurich, Switzerland, ${ }^{4}$ Department of Medicine, University of Vermont, Burlington, VT, United States, ${ }^{5}$ Retired, Underhill, VT, United States, ${ }^{6}$ Department of Reproductive Endocrinology, University of Zurich, Zurich, Switzerland
\end{abstract}

Background: The importance of menstrual cycle physiology in appetite and obesity is poorly understood. We investigated the effects of body mass index (BMI), menstrual cycle phase and sweet and salty taste on monetary valuation of snack foods.

Methods: We recruited 72 women and after the application of in- and exclusion criteria 31 participants with healthy weight and 25 with obesity remained. The participants completed a willingness to pay (WTP) task to measure subjective value of 30 snack food items in the pre-ovulatory and mid-luteal cycle phases.

Results: Generalized linear mixed model (GLMM) analysis revealed that BMl, cycle phase and snack taste interacted to influence WTP $(-0.15[-0.22,-0.03], p=0.002)$. Hence, WTP was inversely related to BMI, but the strength of the relation depended on cycle phase and taste. The WTP of participants with healthy weight for salty taste changed across cycle phase but the WTP for sweet taste was not affected by cycle phase. Moreover, the cycle effect for the salty snacks ceased in participants with obesity.

Conclusion: The inverse effect of BMI on WTP valuation of snack foods contrasts with the positive effect of $\mathrm{BMI}$ on pleasantness ratings for milkshakes by the same women that we previously reported. This indicates that the two measures reflect different aspects of food-related valuative processing in obesity. Furthermore, the WTP data suggest that the selection of salty snacks may differ from that of sweet snacks in the pre-ovulatory phase of the menstrual cycle for individuals of healthy weight. The cycle phase does not seem to affect food valuation of participants with obesity. These findings are relevant to understanding and treating obesity in women.

Keywords: obesity, value-based decision making, food valuation, willingness to pay, ovarian hormones, menstrual cycle

\section{INTRODUCTION}

Obesity remains a pressing issue worldwide. In many countries, obesogenic environments are thought to contribute substantially to obesity's high incidence. These environments include easily available energy-dense foods, which has brought about a relatively novel way of eating known as snacking (Duffey and Popkin, 2011; Mattes, 2018). The calorie intake from snacks add to the calorie 
intake from main meals, and therefore snacking is associated with a higher body mass index (BMI; kg/m²) (Mattes, 2018).

Nevertheless, despite the wide-ranging academic attention devoted to obesity, the roles of reproductive hormones in obesity in women have not often been taken into account. This is important because more women than men suffer from obesity worldwide (NCD Risk Factor Collaboration, 2016). Emotional eating, which is frequently associated with obesity, seems most frequent in the mid-luteal phase of the menstrual cycle and to be influenced by increased levels of both progesterone and $\beta$-estradiol (Klump et al., 2013). Indeed, many studies have shown increased subjective appetite and food intake in the midluteal compared to the mid- to late-follicular and periovulatory phases of the cycle (Buffenstein et al., 1995; Dye and Blundell, 1997; Brennan et al., 2009; Asarian and Geary, 2013; Gorczyca et al., 2016). It is unclear whether these changes are related to altered macronutrient selection. Some studies did not detect changes (Martini et al., 1994; Brennan et al., 2009), whereas others reported increased protein or carbohydrate intake in the mid-luteal phase (Bowen and Grunberg, 1990; Gorczyca et al., 2016). This change in intake could influence the nutrient metabolism in the gut affecting reward signals. In a recent study (de Araujo et al., 2020), the authors suggest that internal signals via the gut-brain-axis generate an unconscious food reward which might constitute the prime driver of overeating. This mechanism is part of a two-roads-to-food-reward model, where food reward is substantially influenced by the flavornutrient learning process happening in the gut (i.e., the lowroad) and results not only from conscious reward signals based on food perception, taste and oral somatosensation (the highroad). Furthermore, a number of differences in taste perception have been linked to obesity and reproductive hormone status (Asarian and Geary, 2013; Hardikar et al., 2017). These studies indicate that better understanding of the effects of reproductive physiology and food type are important for continued progress in obesity research.

Ovarian hormones may affect food intake by changing the motivation to eat. For example, in ovariectomized rats, administration of $\beta$-estradiol resulted in decreased motivation for sucrose rewards (Richard et al., 2017). Such data suggest that estrogens and other ovarian hormones might modulate value-based decision making in relation to food. In addition, diet-induced obesity of female rats is associated with decreased dopamine signaling independent of the menstrual cycle in the nucleus accumbens, which is a fundamental brain region for reward processing (Geiger et al., 2009). We previously analyzed perceived pleasantness of milkshakes in relation to patterns of brain activity detected with fMRI in women with obesity or healthy weight (Gobbi et al., 2020). In that study there was an apparent effect of menstrual cycle phase on perceived pleasantness of sweet milkshakes, but this did not reach statistical significance (Gobbi et al., 2020). Here we expand on these findings studying in the same group of women another aspect of appetite, food valuation as an integration of hunger signals, past experience of the food's flavor and its gastrointestinal and metabolomics effects. To this aim, we used the willingness to pay (WTP) paradigm. The WTP measures the motivation to eat using a bidding and auction method which was developed to quantify an option's utility (Becker et al., 1964). In this study, we aimed to determine if women's valuation of snack foods, first, varies between women with obesity and women with healthy weight, second, varies between the pre-ovulatory and mid-luteal phases of the menstrual cycle, and, third, whether these effects are similar for sweet and salty snack foods.

\section{MATERIALS AND METHODS}

All procedures were approved by the Ethics Commission of the Canton of Zurich, and the participants gave written informed consent. They were compensated for expenditures associated with study participation and received 500 Swiss Francs (CHF) for completing the study.

\section{Participants}

The participants were recruited and screened for eligibility at the department of Reproductive Endocrinology (University Hospital Zurich). In the pre-screening phase, general, endocrinologic and mental health were assessed through a Clinical Assessment Questionnaire designed for the present study. Only individuals fulfilling our previously described (Gobbi et al., 2020) in- and exclusion criteria were recruited. Women with healthy weight (BMI $18.5-24.9 \mathrm{~kg} / \mathrm{m}^{2}$ ) or with grade 1 or 2 obesity (BMI 30$39.9 \mathrm{~kg} / \mathrm{m}^{2}$ ) and who reported that they were weight stable and not dieting were invited to participate. Eligible women were provided with the Three-Factor Eating Questionnaire (TFEQ) and the Eating Disorder Examination-Questionnaire (EDE-Q) to complete at home before the first test visit and to return by mail.

We recruited 72 physically and psychiatrically healthy women who were cycling normally and not using hormonal contraception. Four women withdrew from the study prior to completion, one was excluded due to insufficient German language skills, and two were excluded because of technical difficulties with the tasks. The participants were invited to attend two test visits, one in the pre-ovulatory phase and one in the mid-luteal phase of the menstrual cycle, with order randomized. Tests were timed in relation to menstrual cycle phases, calculated based on cycle information obtained from the participants (i.e., cycle length and days since the last menstruation) and confirmed against progesterone levels. For this paper, the pre-ovulatory phase targeted measurements from $16 \pm 2$ days prior to menstruation, i.e., shortly before ovulation, with low progesterone levels $(3.0 \pm 7.9 \mathrm{nmol} / \mathrm{l})$. The mid-luteal phase targeted $7 \pm 3$ days prior to menstruation with high progesterone levels (21.4 $\pm 18.5 \mathrm{nmol} / \mathrm{l})$. Six participants were excluded because their blood hormone levels were not in agreement with calculated cycle phases in either cycle phase. Another six participants for which only one cycle phase could be confirmed were retained, with their missing data accounted for in the statistical analyses. Three participants were excluded because their 
mean cycle length was not within the normal range (2535 days).

\section{Test Procedure}

Participants were advised not to drink or eat anything after $10 \mathrm{pm}$ on the evening prior to both test visits. The study compensation fee served as credit for the WTP task. In this task, participants bid money on a scale from 0 to $2.5 \mathrm{CHF}$ in accordance with their desire to obtain different food items, which were pictures of 15 sweet and 15 salty common snack foods displaying a serving size of the product in front of its package on a black background. The snacks predominantly comprised chocolate bars, nut bars, cookies, gummy bears, crackers, olives, crisps, and salted nuts. To account for potential successive contrast influences among food items, this procedure was done twice for each food item in different order, resulting in 60 WTP bids. The participants bid on a continuous scale using a trackball, which they moved to the desired bid amount and then pressed to confirm their choice. The compensation method for the task was determined by an incentive-compatible auction mechanism (Becker et al., 1964). At the end of each session, one trial was selected at random and implemented. If the participant's bid for the food item in that trial was greater than or equal to the auction price, they paid the auction price from their compensation fee and they received the snack to consume at the laboratory, before leaving. Otherwise, the participant did not obtain the food item and kept the entire compensation amount. The WTP task and several other tasks were completed whilst lying supine in a whole-body MRI scanner [Philips Medical Systems, Laboratory for Social and Neural Systems research (SNS Lab) in Zürich University Hospital, Zürich, Switzerland; more details regarding the scanning procedure and the other experiments are described elsewhere (Gobbi et al., 2020)]. In addition to the WTP task reported here, participants performed a task assessing food value measured by willingness to exert physical effort and a task related to experienced food value (milkshake sampling), which was previously reported (Gobbi et al., 2020). Trials of the different tasks were presented in random order. In addition, on each test day, participants performed the tasks in the MRI twice, once before and once after an ad libitum meal. The meal consisted of ham sandwiches and tap water, and the consumed amount was determined by comparing the weight of sandwiches before and after the meal. Weights were transformed to kcal and analyzed as ad libitum consumption level. Participants had $30 \mathrm{~min}$ to finish the meal and then returned to the scanner and repeated the three valuation tasks. Hence, the participant performed the tasks in two satiety states (fasted, fed) and two menstrual cycle phases (pre-ovulatory, mid-luteal), resulting in four sessions in total.

At different time points throughout the experiment, participants rated a number of subjective states. These included hunger, satiety, desire to eat, nausea, tiredness, feeling well, anxiety, discomfort, agitation, and dizziness. The participants provided these ratings using a generalized visual analog scale ranging from "not at all" to "as strong as possible" (Blundell et al., 2010). The participants answered by moving a trackball along the rating scale and clicking to indicate their response. For both WTP and subjective state ratings the scale direction was randomized across trials and sessions to disentangle brain activation from physical skills or habits related to moving the trackball.

\section{Statistical Analysis}

Statistical analyses were performed using Microsoft Excel 2013 and $\mathrm{R}$ version 4.0.2 ( $\mathrm{R}$ Core Team, 2013). Participants were weighed during both visits, which resulted in two different BMIs per participant $\left(\mathrm{kg} / \mathrm{m}^{2}\right)$. For the Spearman correlation analyses and the generalized linear mixed model (GLMM) analysis, BMI was treated as a continuous variable, whereas for group comparisons, we used the categorical variable weight status, i.e., healthy weight or obese.

Willingness to pay bids without confirmation (no trackball press) were considered invalid, i.e., entered the analysis as missing values. In addition, we excluded items with WTP bids of zero in all four experimental sessions due to an apparent dislike of these items. Visual inspection of the histograms and the quantilequantile plots indicated that the WTP bids and several other dependent variables were not normally distributed. Therefore, non-parametric statistical analyses were done for all analysis except the GLMM, which was based on a beta distribution. We computed Spearman correlation coefficients $\left(r_{s}\right)$ for WTP bids, BMI, ad libitum consumption levels, TFEQ scores, and the subjective state ratings from the time points around the WTP task, excluding missing values. A correlation matrix (Figure 1) was constructed using the R function corrplot (Wei et al., 2017). Magnitudes of the correlations are reported with the Spearman correlation coefficient $\mathrm{r}_{s}$.

We also contrasted the mean WTP bids, the EDE-Q and the TFEQ scores of the groups with healthy weight and obesity using Wilcoxon signed-rank tests for independent samples and we conducted group comparisons for WTP differences for sweet or salty food items using Wilcoxon signed-rank tests for paired samples. To calculate effect sizes $r$ for the Wilcoxon tests we used the R function wilcox_effsize (Kassambara, 2020). We further tested for mean differences in WTP across cycle phases. To account for seven participants who completed two test visits in the same cycle phase and six participants for whom only one cycle phase was confirmed, we applied a method designed for paired data with missing values (Fong et al., 2018) using the $\mathrm{R}$ function pm.wilcox.test with the SR-MW method, which consists of a combination of signed-rank statistics for paired data and Mann-Whitney statistics for unpaired data (Fong et al., 2018). Because this test results in a $Z$-value, the effect sizes were calculated as the $Z$-statistic divided by the square root of the number of observations (Rosenthal, 1994). Visualizations of group comparisons were obtained using ggplot (Wickham, 2006). The Spearman correlations and group contrasts were considered exploratory, so we used a significance level of $\mathrm{a}=0.05$.

The dependent variable for the GLMM analysis was the WTP. As these data followed an extreme values distribution determined by the monetary rating boundaries of 0 and 2.5 (in CHF), we used a beta regression model and a logit function as link (Cribari-Neto and Zeileis, 2010). The data were divided by 2.5 to normalize WTP values $\left(\mathrm{WTP}_{\text {Norm }}\right.$ ) to between 0 and 1 , as previously suggested (Smithson and Verkuilen, 2006), and transformed using the function $\mathrm{WTP}_{\text {conv }}=\left(\left[\mathrm{WTP}_{\text {Norm }}(\mathrm{n}-1)+0.5\right] / \mathrm{n}\right)$ where 


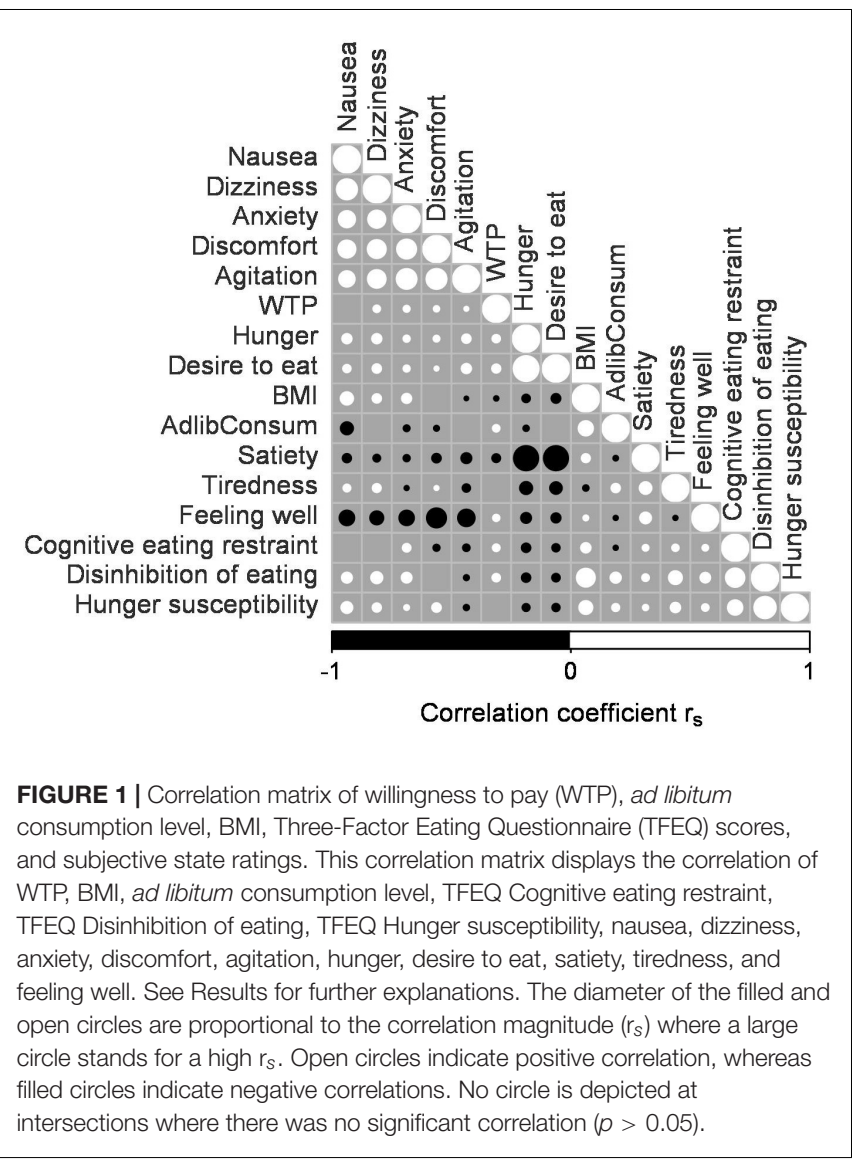

$\mathrm{n}$ is the number of samples (Smithson and Verkuilen, 2006). We used the glmmTMB R package because it enables inclusion of random effects in the model (Brooks et al., 2017). We included random intercepts for participants and items as well as participant-specific random slopes for Day and Satiety. The model is defined as follows:

$$
\begin{gathered}
\text { WTP }_{i j}=\left(\beta_{0}+u_{0 j}+u_{0 k}\right)+\left(\beta_{1}+u_{1 j}\right) \text { Day }_{i j}+\left(\beta_{2}+u_{2 j}\right) \\
\text { Satiety }_{i j}+\beta_{3} C P_{i j}+\beta_{4} \text { AdlibConsum }_{i j}+\beta_{5} B M I_{i j}+\beta_{6}
\end{gathered}
$$$$
\text { Taste }_{i j}+\beta_{7} \text { AdLibConsum }_{i j} B M I_{i j}+\beta_{8} C_{i j} \text { AdLibConsum }_{i j}+
$$$$
\beta_{9} C P_{i j} B M I_{i j}+\beta_{10} B M I_{i j} \text { Taste }_{i j}+\beta_{11} C P_{i j} \text { Taste }_{i j}+
$$$$
\beta_{12} \text { AdLibConsum }_{i j} \text { Taste }_{i j}+\beta_{13} \text { CP }_{i j} \text { AdLibConsum }_{i j} B M I_{i j}+
$$$$
\beta_{14} C P_{i j} \text { BMI }_{i j} \text { Taste }_{i j}+\beta_{15} \text { CP }_{i j} \text { AdLibConsum }_{i j} \text { Taste }_{i j}+e_{i j}
$$

The index $\mathrm{i}$ represents the trial, $\mathrm{j}$ the participant and $\mathrm{k}$ the item. The continuous variables BMI and ad libitum consumption level (AdlibConsum) were $z$-scored at a group level and day of test visit (Day; Day 1 or Day 2), Satiety (Fasted or Fed), cycle phase (CP; Pre-ovulatory or Mid-luteal), and Taste (Sweet or Salty) were used as binary variables. Two- and three-way interactions were included. We calculated the $p$-values using the Wald statistic. To correct for the multiple tests using WTP bids as dependent variable (correlation, three Wilcoxon tests and the GLMM), we applied the Bonferroni procedure, resulting in a nominal threshold of $0.01(=0.05 / 5)$, and only effects for which $p$-value $<0.01$ were considered significant. To display significant interaction effects we used the $\mathrm{R}$ functions ggpredict and ggplot (Wickham, 2006; Lüdecke, 2018).

\section{RESULTS}

\section{Subjects}

We report the results for 56 women who fulfilled our selection criteria. Table 1 shows the participants' demographic data. We assessed the cycle phases more conservatively than previously, resulting in fewer participants than in our previous paper (Gobbi et al., 2020). Forty-six (82\%) women submitted complete questionnaires. These participants' global EDE-Q scores were $1.4 \pm 1.1$, which closely matches community norm values (Fairburn et al., 2014). The EDE-Q scores Restraint, Eating concern, Shape concern and Weight concern were all significantly lower for participants with healthy weight than for those with obesity (median Restraint 0.4 and 1.2, $r=0.36, p=0.016$; Eating concern 0.2 and $0.8, r=0.51, p<0.001$; Weight concern 0.1 and 3.0, $r=0.68, p<0.001$; and Shape concern 1.1 and 3.5, $r=0.65, p<0.001)$. The TFEQ scores cognitive eating restraint and hunger susceptibility did not differ between participants with healthy weight and with obesity $(p=0.414$ and $p=0.058)$ but disinhibition of eating was higher in the obese than in the healthy weight group (median 9.0 and 7.0, $r=0.40, p=0.007$ ).

\section{Spearman Correlations}

Figure 1 summarizes the Spearman correlational data. The WTP bids correlated very weakly positively with desire to eat, hunger, feeling well, dizziness, anxiety, agitation and discomfort $\left(r_{s} \leq 0.1\right.$, $p \leq 0.011)$ and negatively with satiety rating $\left(r_{s}=-0.09\right.$, $p<0.001)$ and BMI $\left(r_{s}=-0.03, p=0.002\right)$. Analysis of the TFEQ scores revealed a weak but significant correlation of BMI with cognitive eating restraint and hunger susceptibility $\left(r_{s}=0.13\right.$, $p<0.001$ and $\left.r_{s}=0.25, p<0.001\right)$ and a moderate correlation of BMI with disinhibition of eating $\left(r_{s}=0.46, p<0.001\right)$. We also found very weak correlations of WTP with cognitive restraint and with disinhibition of eating $\left(r_{s} \leq 0.1, p<0.001\right.$; Figure 1). Hence, the Spearman correlations of WTP bids with the subjective state ratings, TFEQ scores and ad libitum consumption level failed to reveal any strong relationships.

\section{Group Comparisons}

The WTP bids (in CHF) of participants with healthy weight were significantly reduced in the pre-ovulatory compared to the mid-luteal phase (median 0.95 and 1.10, $r=-0.14, p<0.001$ ), whereas those of women with obesity did not change significantly across cycle phases ( $p=0.334$; Figure 2). Furthermore, group comparisons within cycle phase showed that the pre-ovulatory WTP bids were higher in participants with obesity than in 
TABLE 1 | Demographics of the participants fulfilling the inclusion criteria.

\begin{tabular}{|c|c|c|}
\hline & Mean \pm SD & Range \\
\hline Age $(y)$ & $25.5 \pm 4.7$ & $(18-40)$ \\
\hline Healthy weight $(n=31)$ & $26.0 \pm 5.0$ & $(19-40)$ \\
\hline Obese $(n=25)$ & $24.9 \pm 4.4$ & $(18-33)$ \\
\hline $\mathrm{BMI}\left(\mathrm{kg} / \mathrm{m}^{2}\right)$ & $26.9 \pm 5.4$ & $(18.8-37.4)$ \\
\hline Healthy weight $(n=31)$ & $22.3 \pm 2.1$ & $(18.8-25.9)$ \\
\hline Obese $(n=25)$ & $32.3 \pm 2.2$ & $(29.0-37.4)$ \\
\hline Cycle length (d) & $29 \pm 2$ & $(25-35)$ \\
\hline Healthy weight $(n=31)$ & $28 \pm 2$ & $(25-32)$ \\
\hline Obese $(n=25)$ & $29 \pm 2$ & $(27-35)$ \\
\hline EDE-Q: restraint & $1.0 \pm 1.0$ & $(0-3.6)$ \\
\hline Healthy weight $(n=27)$ & $0.6 \pm 0.8$ & $(0-3.2)$ \\
\hline Obese $(n=19)$ & $1.4 \pm 1.2$ & $(0-3.6)$ \\
\hline EDE-Q: eating concern & $0.6 \pm 0.8$ & $(0-3.0)$ \\
\hline Healthy weight $(n=27)$ & $0.3 \pm 0.4$ & $(0-1.4)$ \\
\hline Obese $(n=19)$ & $1.1 \pm 1.0$ & $(0-3.0)$ \\
\hline EDE-Q: shape concern & $2.1 \pm 1.5$ & $(0-5.4)$ \\
\hline Healthy weight $(n=27)$ & $1.3 \pm 0.8$ & $(0-3.6)$ \\
\hline Obese $(n=19)$ & $3.3 \pm 1.4$ & $(0.8-5.4)$ \\
\hline EDE-Q: weight concern & $1.8 \pm 1.5$ & $(0-5.2)$ \\
\hline Healthy weight $(n=27)$ & $0.9 \pm 0.7$ & $(0-2.4)$ \\
\hline Obese $(n=19)$ & $3.0 \pm 1.4$ & $(0.4-5.2)$ \\
\hline TFEQ: cognitive eating restraint & $6.9 \pm 4.0$ & $(1-15)$ \\
\hline Healthy weight ( $n=27$ ) & $6.5 \pm 4.4$ & $(1-14)$ \\
\hline Obese $(n=19)$ & $7.5 \pm 3.5$ & $(2-15)$ \\
\hline TFEQ: disinhibition of eating & $7.1 \pm 3.1$ & $(1-15)$ \\
\hline Healthy weight $(n=27)$ & $5.9 \pm 3.2$ & $(1-11)$ \\
\hline Obese $(n=19)$ & $8.7 \pm 3.5$ & $(2-15)$ \\
\hline TFEQ: hunger susceptibility & $5.3 \pm 3.6$ & $(0-13)$ \\
\hline Healthy weight $(n=27)$ & $4.6 \pm 2.9$ & $(0-11)$ \\
\hline Obese $(n=19)$ & $6.3 \pm 3.1$ & $(1-13)$ \\
\hline
\end{tabular}

Data are mean $\pm S D$ and range for all 56 participants except as noted. BMI [weight $(\mathrm{kg}) /$ height $^{2}\left(\mathrm{~m}^{2}\right)$ ] are the data collected on the two test days. The women with healthy weight include three women whose BMI increased from below 25 at screening to between 25 and 26 during the tests, and the women with obesity include three women whose BMI decreased from above 30 at screening to between 29 and 30 during the tests.

participants with healthy weight (median 1.00 and $0.95, r=0.05$, $p=0.012$ ) whereas the mid-luteal WTP bids were lower in participants with obesity than those with healthy weight (median 1.00 and $1.10, r=0.07, p<0.001)$. Thus, obesity eliminated the effect of the menstrual cycle on WTP for snack foods that was evident in women with healthy weight (Figure 2).

On average, the WTP bids for sweet snacks were higher than the ones for salty snacks (median 1.08 and $0.98, r=0.13$, $p<0.001$ ). This difference arose in both cycle phases (preovulatory $r=0.14, p=0.002$ and mid-luteal $r=0.12, p=0.010$ ) and weight groups (healthy weight $r=0.12, p=0.010$ and obese $r=0.15, p=0.002$ ). Hence, WTP differed depending on the taste of the snack foods presented, i.e., sweet or salty.

\section{Generalized Linear Mixed Model}

The GLMM outcomes are summarized in Table 2. The satiety state significantly affected $\mathrm{WTP}_{\text {conv }}\left(p_{\text {corrected }}<0.01\right)$.
Specifically, average $\mathrm{WTP}_{\text {conv }}$ was higher in fasted compared to fed satiety state $(Z=-5.13, p<0.001)$. The predictor snack type ("taste") showed a trend-level effect but did not survive Bonferroni correction. There were two significant interactions predicting $\mathrm{WTP}_{\text {conv }}$ bids $\left(p_{\text {corrected }}<0.01\right)$. The first interaction was between snack type and cycle phase $(Z=4.27, p<0.001)$, the second one between snack type, cycle phase and BMI $(Z=-3.07$, $p=0.002)$. As shown in Figure 3, $\mathrm{WTP}_{\text {conv }}$ was inversely related to BMI but the strength of the relation depended on cycle phase and snack type. That is, for both snack types in the mid-luteal phase and for sweet snacks in the pre-ovulatory phase, mean $\mathrm{WTP}_{\text {conv }}$ decreased similarly from $\sim 0.50$ in participants with the lowest BMI to $\sim 0.35$ in participants with the highest BMI. In contrast, $\mathrm{WTP}_{\text {conv }}$ for salty snacks in the pre-ovulatory phase was less, $\sim 0.41$, in participants with the lowest BMI, and decreased less steeply, to $\sim 0.37$ for participants with the highest BMI. Furthermore, WTP bids for salty foods were generally higher in women with lower BMI than with high BMI, especially during the mid-luteal phase. In contrast, for sweet snacks, WTP bids were also generally higher in women with lower BMI, but were not affected by menstrual cycle phase.

\section{DISCUSSION}

\section{Food Valuation Depends on Obesity, Cycle Phase, and Snack Taste}

In this study we used a WTP method to measure the subjective value of snack foods in women with healthy weight or obesity. We report an inverse relationship between WTP and BMI, which depends on snack taste and cycle phase. The decreasing WTP with increasing BMI is opposite to what we found in a milkshake tasting task, where the same participants' pleasantness ratings increased with BMI (Gobbi et al., 2020). This indicates that the different tasks link to different parts of the reward and valuation systems. Specifically, rating milkshake pleasantness is a more sensory or consummatory process, whereas WTP is a more anticipatory process. Moreover, compared to typical anticipatory ratings, such as expected satiety (Brunstrom, 2014), the WTP task is a more complex decision-making and valueestimation task. Indeed, during the milkshake task our fMRI data indicated that predominantly hedonic and homeostatic brain circuits were activated (Gobbi et al., 2020) whereas WTP has been reported to be associated with activity in different regions of the prefrontal cortex (Plassmann et al., 2007; Tang et al., 2014). This might reflect involvement of cognition and value computation in the WTP task, which is in line with the prefrontal cortex being involved in cognitive control of goal-directed behavior (Miller and Cohen, 2001). Relatedly, cognitive restraint of eating may have influenced WTP bidding of participants with obesity. High cognitive restraint is positively associated with BMI [e.g., Banna et al. (2018) and Adams et al. (2019)] and has been reported to dampen cyclic eating changes (Asarian and Geary, 2013). We measured a small positive correlation of both WTP and BMI with the TFEQ score cognitive eating restraint and the EDE-Q scores Restraint and Eating concern were 


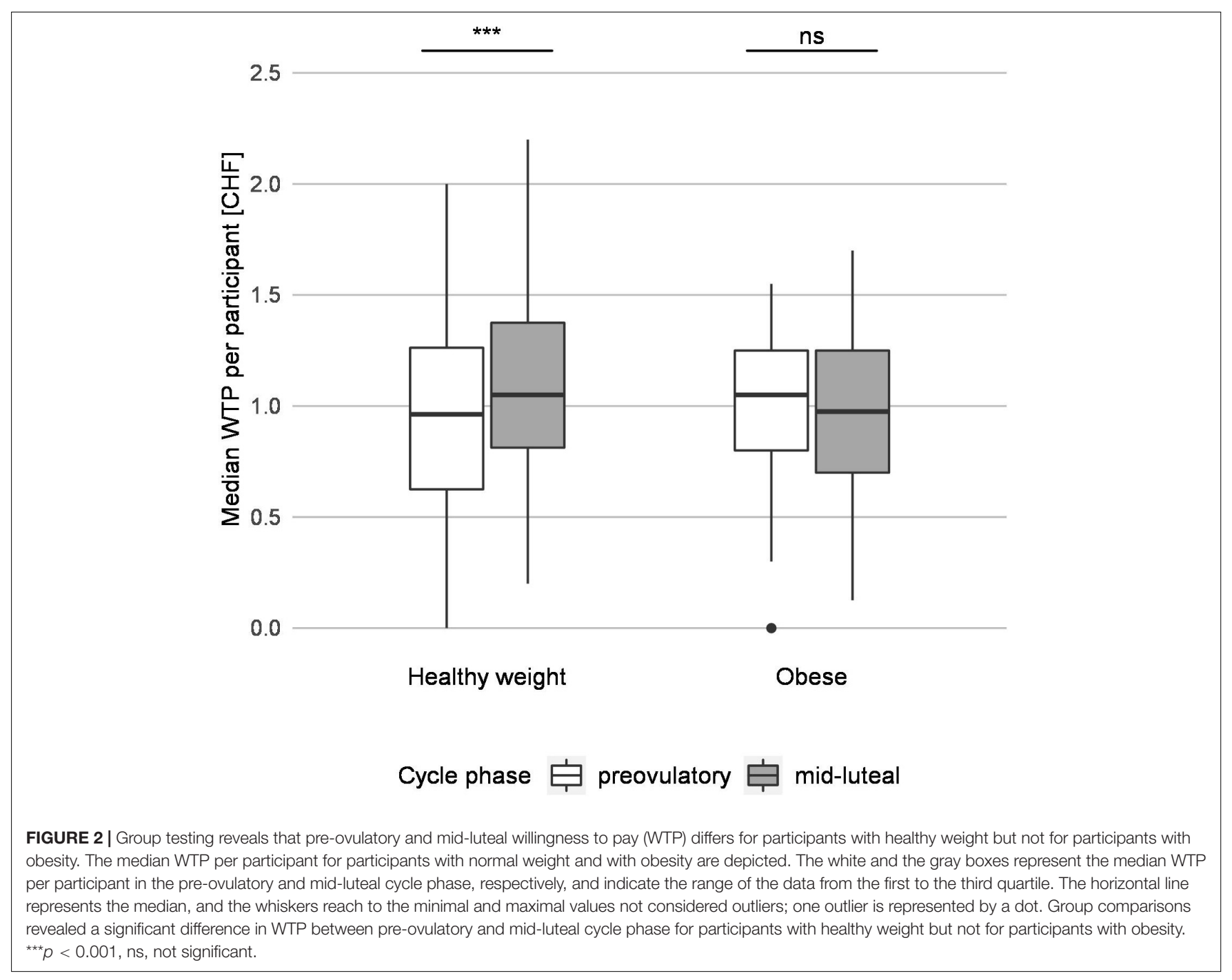

significantly increased in women with obesity. Hence, WTP of participants with obesity might be lower in part due to cognitive restraint.

\section{Food Valuation Differs by Cycle Phase for Salty Snacks}

Our findings reveal that valuation appears to be higher for sweet than for salty snack foods, especially for participants with obesity and in the pre-ovulatory cycle phase. In contrast, mid-luteal WTP bids of participants with healthy weight did not differ by taste. Hence, while the WTP for salty snacks increased from the pre-ovulatory to mid-luteal cycle phase, the WTP for sweet snacks did not depend on cycle phase. This absence of cycle effect for sweet food parallels the findings on pleasantness of milkshakes (Gobbi et al., 2020). The mid-luteal increase in women's value for salty food is in accordance with an apparent stronger dislike of unsalted popcorn in the luteal phase compared to the follicular phase (Frye and Demolar, 1994). However, neither intake of nor preference ratings for salty food changed between menstrual cycle phases (Bowen and Grunberg, 1990). Thus, further research is required to assess the relationship between the valuation change and actual intake of salty foods in snack and non-snack contexts. We conclude, first, that women's food valuation differs by taste and, second, that valuation for salty food but not for sweet food differs by cycle phase.

\section{Possible Mechanisms}

Previous neuroimaging studies demonstrated correlations between WTP and activity of different brain regions (Plassmann et al., 2007; Tang et al., 2014). Some of these are associated with subjective values, reinforcing the idea that the WTP approach is a valid measure of subjective value (Bartra et al., 2013). We found that WTP is lower in the pre-ovulatory than in the mid-luteal phase for participants with healthy weight. In line with this, another study found that the appeal of food images was lower in the second week of the menstrual cycle than in the last week (Frank et al., 2010). Such changes across the menstrual cycle have also been reported for brain activation in response to food images and uncertain monetary rewards 
TABLE 2 | The generalized linear mixed model (GLMM) reveals a main effect of satiety state, an interaction of cycle phase with snack taste, and a three-way interaction of cycle phase with snack taste and BMI to significantly predict WTP conv $_{\text {. }}$

\begin{tabular}{|c|c|c|c|c|}
\hline & Estimate & SE & Z-statistic & $P$-value \\
\hline (Intercept) & $-0.15[-0.34,0.09]$ & 0.14 & -1.12 & 0.264 \\
\hline Mid-luteal & $-0.04[-0.21,0.15]$ & 0.09 & -0.40 & 0.687 \\
\hline AdlibConsum & $0.08[-0.11,0.25]$ & 0.09 & 0.86 & 0.391 \\
\hline BMI & $-0.15[-0.33,0.06]$ & 0.10 & -1.45 & 0.146 \\
\hline Salty & $-0.29[-0.36,-0.23]$ & 0.12 & -2.37 & 0.018 \\
\hline Fed & $-0.24[-0.32,-0.14]$ & 0.05 & -5.13 & $<0.001 *$ \\
\hline Day 2 & $-0.03[-0.18,0.13]$ & 0.08 & -0.36 & 0.717 \\
\hline AdlibConsum $\times$ BMI & $0.05[-0.11,0.22]$ & 0.08 & 0.65 & 0.515 \\
\hline Mid-luteal $\times$ AdlibConsum & $0.10[-0.09,0.30]$ & 0.10 & 1.00 & 0.319 \\
\hline Mid-luteal × BMI & $0.01[-0.18,0.18]$ & 0.09 & 0.15 & 0.878 \\
\hline BMI $\times$ Salty & $0.08[-0.01,0.13]$ & 0.04 & 2.21 & 0.027 \\
\hline Mid-luteal $\times$ Salty & $0.20[0.09,0.28]$ & 0.05 & 4.27 & $<0.001^{\star}$ \\
\hline AdlibConsum $\times$ Salty & $-0.04[-0.11,0.04]$ & 0.04 & -1.12 & 0.263 \\
\hline Mid-luteal $\times$ AdlibConsum $\times$ BMl & $-0.07[-0.25,0.10]$ & 0.09 & -0.73 & 0.466 \\
\hline Mid-luteal $\times$ BMI $\times$ Salty & $-0.15[-0.22,-0.03]$ & 0.05 & -3.07 & $0.002^{\star}$ \\
\hline Mid-luteal $\times$ AdlibConsum $\times$ Salty & $-0.05[-0.16,0.04]$ & 0.05 & -1.03 & 0.303 \\
\hline Number of observations: & \multicolumn{4}{|c|}{11,022} \\
\hline BIC: & \multicolumn{4}{|c|}{-14655.2} \\
\hline
\end{tabular}

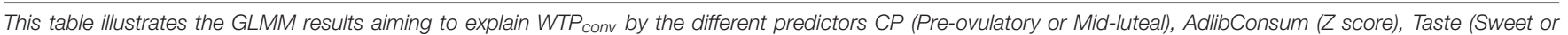

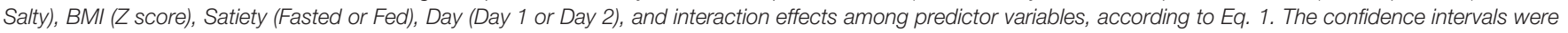

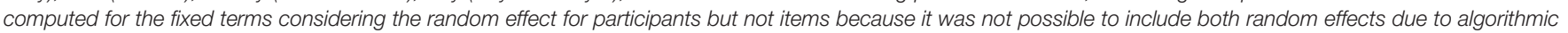

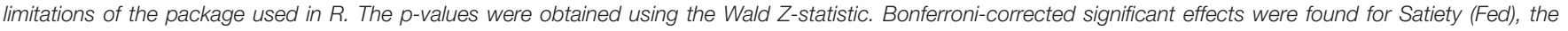

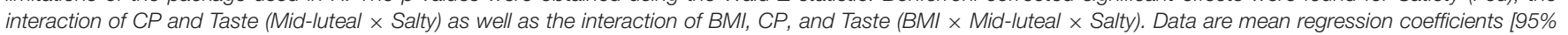
$\mathrm{Cl}$ ] and all continuous regressors were $z$-scored before entering the model. P-values are uncorrected; *Significant following Bonferroni-correction, $p_{\text {corrected }<0.01 .}$

(Dreher et al., 2007; Frank et al., 2010). Furthermore, rat studies have revealed that estrogens enhance striatal dopamine signaling (Becker and Cha, 1989; Becker, 1999; Yoest et al., 2014). Although neither estrogens nor progestins are sufficient to explain increased energy intakes in the mid-luteal cycle phase in women or other anthropoid primates (Asarian and Geary, 2013), increases in the two hormones have been associated with increases in emotional eating during the mid-luteal phase (Klump et al., 2013) and progesterone and $\beta$-estradiol synergized to induce striatal dopamine release in rats (Yoest et al., 2018).

As described above, WTP bids decreased with increasing BMI, but changed across the menstrual cycle only in participants with healthy weight bidding for salty snacks. At least the former effect may be related to dopamine signaling. The reward behavior in the group with obesity, which did not depend on the menstrual cycle, supports results of experiments with female rats which showed that an obesity-inducing regimen decreased mesolimbic dopamine transmission (Geiger et al., 2009). Furthermore, reduced activation of reward-associated brain regions in individuals with obesity has been shown in human trials (Carnell et al., 2012), and striatal dopamine $\mathrm{D}_{2}$ receptor expression was lower in individuals with severe obesity compared to humans with healthy weight (Wang et al., 2001). Relatedly, long term intake of energy-rich food (Burger and Stice, 2011) and high saturated-fat diets (Kleinridders and Pothos, 2019) decrease dopamine signaling in reward-associated brain areas. Thus, chronic downregulation of dopamine in certain brain regions of individuals with obesity may interfere with reward processing, which would otherwise depend on menstrual cycle. Further research should address how dopamine signaling affects valuation of different foods across the menstrual cycle and in women with different BMI.

\section{Subjective State Ratings and Satiety State}

The GLMM revealed a significant main effect of satiety state (fed or fasted) as a predictor for $\mathrm{WTP}_{\text {conv. This supports }}$ theoretical approaches suggesting that value-based decision making depends on hunger (Niv et al., 2006; Rangel et al., 2008) and that the food evaluation process is influenced by the gut-brain-axis (de Araujo et al., 2020). In apparent contrast, our correlational analyses suggested that WTP bids were not substantially related to subjective states. In view of the interactive effect of BMI, cycle phase and snack taste revealed by the GLMM, however, it is not surprising that simple correlational analyses failed. The lower power of nonparametric correlations may have also contributed. It is also possible that subjective ratings are weaker measures of food valuation than our behavioral and fMRI measures. For example, the weak correlation of satiety ratings and WTP contrasts with the clear main effect of satiety state as a predictor for

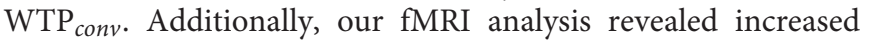
striatal and prefrontal activity in participants with obesity 


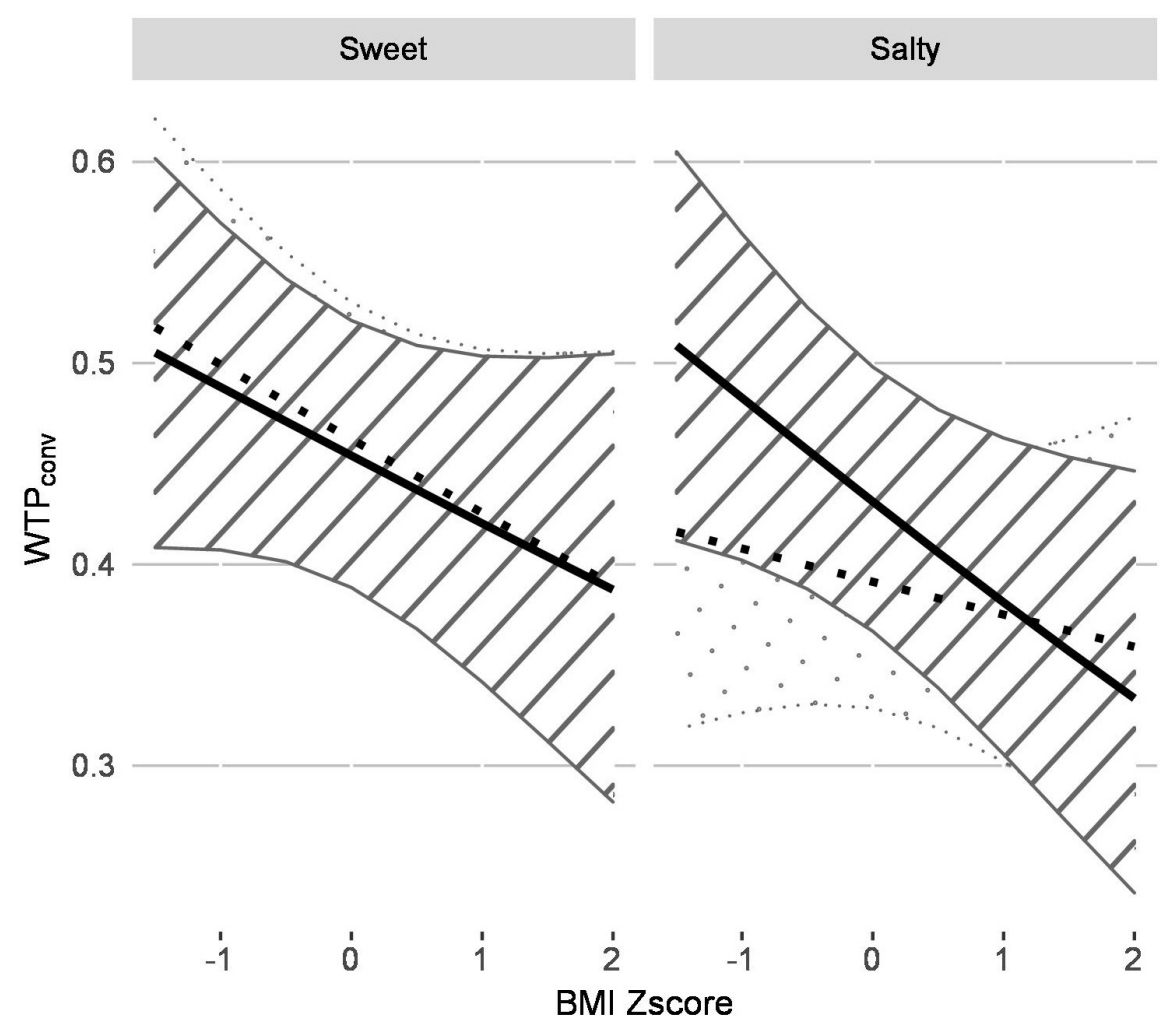

Cycle Phase $\because$ preovulatory

FIGURE 3 | The generalized linear mixed model (GLMM) reveals that the relationship between WTP conv is inversely related to BMI for both sweet and salty snacks and menstrual cycling affects WTP conv for salty, but not for sweet, snacks. The plot visualizes how cycle phase, taste and BMI interact to predict WTP conv $_{\text {according }}$ to the GLMM. WTP conv is inversely related to BMl and the strength of this relationship depends on cycle phase and taste. For sweet food items, average pre-ovulatory and mid-luteal WTP conv both decrease with increasing BMI and the GLMM only predicted a slight difference between cycle phases. For salty food items, higher average WTP bids are predicted in the mid-luteal compared to the pre-ovulatory cycle phase for participants with low BMI whereas this effect diminishes with increasing BMI. Control analyses verified that the overall effect was reflected in the data of the individual groups.

despite their ratings of high satiety and low desire to eat (Gobbi et al., 2020).

\section{Strengths and Limitations}

We used BMI as a metric to discriminate between people with and without obesity rather than other methods like dual energy $\mathrm{x}$-ray absorptiometry (DXA) because it remains a simple and accurate measure for obesity on a population level (The World Health Organization [WHO], 2022). However, the association between BMI and body fat content is limited because BMI does not consider age, physical activity level, and sex (The World Health Organization [WHO], 2022). Still, in our study, we controlled for the variables age and sex as only adult, premenopausal women were included. Moreover, we asked the participants to fill out the International Physical Activity Questionnaire (IPAQ) to determine their physical activity (Craig et al., 2013) because it has been shown that BMI can be especially inaccurate for individuals with a high muscle mass, e.g., athletes. Indeed, the literature suggests that athletes' food choices are influenced not only by the commonly found factors taste, convenience, and nutrition (Birkenhead and Slater, 2015). Sport requires a different type of energy supply and athletes choose food in order to optimize their performance (Parraga, 1990; Eertmans et al., 2005; Birkenhead and Slater, 2015). Likewise, weight-conscious individuals usually prioritize low-energy foods that are helpful for their diet and body composition compared to more palatable choices (Birkenhead and Slater, 2015). Thus, we could expect sports involvement to enhance inhibitory control and dietary self-control (Wills et al., 2007; Lowe et al., 2016) and consequentially to decrease the positive evaluation of food snacks in the WTP task implemented in the study. The IPAQ allowed us to control for differences in the level of physical activity between people with obesity and with healthy weight; on average between groups, we did not find any. Therefore, we can exclude high BMI due to increased muscle mass. Furthermore, the difference of body weight in an average Swiss woman (1.64 m tall; Eglitis-media, 2022) between a BMI of 22.3 and 32.3 (mean BMI of the two groups) corresponds to $26.9 \mathrm{~kg}$, which is unlikely to be due to 
differences in muscle mass alone. Hence, in view of these considerable differences of BMI between groups and the control for confounding factors such as age, sex, and physical activity, we considered BMI as a sufficient measure to differentiate participants with healthy weight from those with obesity. In general, our inclusion criteria were rather strict in order to increase the likelihood of detecting causal neuroendocrine effects. Although we invited only participants with a BMI greater than 30 or below 25, some participants changed body weight enough to bring their BMI outside the inclusion criteria. This was unlikely to be of high importance for the majority of our analysis in which BMI was used as a continuous variable. Participants were informed that the study was about steroid hormones and asked to maintain menstrual cycle records; this might have promoted response biases related to their beliefs about menstrual cycle and appetite. Timing may have affected some outcomes because food preferences are lower in the morning, when our experiment took place, than later in the day (Reichenberger et al., 2018). Lastly, other factors, such as prices, social information, habituation and labels, might have influenced WTP ratings in our test situation (List and Shogren, 1999; Niv et al., 2006; Rangel et al., 2008; Suzuki et al., 2017; Motoki and Suzuki, 2020). To minimize habituation bias, we organized the study sessions in random order with respect to cycle phase. Furthermore, the participants were provided with all instructions prior to the test sessions and they conducted the WTP task alone in the scanner.

\section{CONCLUSION}

This study aimed to measure food valuation and to investigate whether it changes with BMI, cycle phase, and taste. Our major findings were that WTP changes across the menstrual cycle for participants with healthy weight and for salty food items. This indicates that obesity research should focus more on the influences of, first, types of foods and eating occasions and, second, reproductive hormones on reward processing, in particular dopamine signaling and anticipatory food valuation. Furthermore, the reduction in the effect of menstrual cycle changes on food valuation in women with obesity is a novel finding which, if reproduced by future studies, may be critical for future obesity research.

\section{REFERENCES}

Adams, R. C., Chambers, C. D., and Lawrence, N. S. (2019). Do restrained eaters show increased BMI, food craving and disinhibited eating? A comparison of the restraint scale and the restrained eating scale of the dutch eating behaviour questionnaire. R. Soc. Open Sci. 6:190174. doi: 10.1098/rsos.19 0174

Asarian, L., and Geary, N. (2013). Sex differences in the physiology of eating. Am. J. Physiol. Regul. Integr. Comp. Physiol. 305, R1215R1267.

Banna, J. C., Panizza, C. E., Boushey, C. J., Delp, E. J., and Lim, E. (2018). Association between cognitive restraint, uncontrolled eating, emotional eating and BMI and the amount of food wasted in early adolescent girls. Nutrients 10:1279. doi: 10.3390/nu10091279

\section{DATA AVAILABILITY STATEMENT}

The datasets generated and/or analyzed during the current study are available from the corresponding author on reasonable request. Requests to access the datasets should be directed to $\mathrm{BL}$, brigitte.leeners@usz.ch.

\section{ETHICS STATEMENT}

The studies involving human participants were reviewed and approved by Ethics Commission of the Canton of Zurich. The patients/participants provided their written informed consent to participate in this study.

\section{AUTHOR CONTRIBUTIONS}

LH planned and performed the data analysis and wrote the first draft of the manuscript. SG collected and curated the data, planned and supported the data analysis. SW programmed the task and collected data. GG, MR, and BL realized recruitment and selected the participants. BL, LA, NG, PT, MR, and SW designed the study. All authors contributed to the manuscript and approved the final version.

\section{FUNDING}

This work was supported by funding from the Marlene Porsche Foundation, the Zurich Center for Integrative Human Physiology, Philhuman Foundation, Foundation for Nutrition Research, Foundation for Scientific Research University of Zurich, Baugarten Foundation, and the Swiss NSF (grants PP00P1 150739 and 100014_165884 to PT).

\section{ACKNOWLEDGMENTS}

We would like to thank the participants from this study, Ferdinand von Meyenn for his valuable comments and discussions, and Daria Hinz and Karl Treiber for their assistance with the data collection.

Bartra, O., McGuire, J. T., and Kable, J. W. (2013). The valuation system: a coordinate-based meta-analysis of BOLD fMRI experiments examining neural correlates of subjective value. Neuroimage 76, 412-427. doi: 10.1016/j. neuroimage.2013.02.063

Becker, G. M., Degroot, M. H., and Marschak, J. (1964). Measuring utility by a single-response sequential method. Behav. Sci. 9, 226-232. doi: 10.1002/bs. 3830090304

Becker, J. B. (1999). Gender differences in dopaminergic function in striatum and nucleus accumbens. Pharmacol. Biochem. Behav. 64, 803-812. doi: 10.1016/ s0091-3057(99)00168-9

Becker, J. B., and Cha, J.-H. (1989). Estrous cycle-dependent variation in amphetamine-induced behaviors and striatal dopamine release assessed with microdialysis. Behav. Brain Res. 35, 117-125. doi: 10.1016/s0166-4328(89) 80112-3 
Birkenhead, K. L., and Slater, G. (2015). A review of factors influencing athletes' food choices. Sports Med. 45, 1511-1522. doi: 10.1007/s40279-015-0372-1

Blundell, J., de Graaf, C., Hulshof, T., Jebb, S., Livingstone, B., Lluch, A., et al. (2010). Appetite control: methodological aspects of the evaluation of foods. Obes. Rev. 11, 251-270. doi: 10.1111/j.1467-789X.2010.00714.x

Bowen, D. J., and Grunberg, N. E. (1990). Variations in food preference and consumption across the menstrual cycle. Physiol. Behav. 47, 287-291. doi: 10. 1016/0031-9384(90)90144-s

Brennan, I. M., Feltrin, K. L., Nair, N. S., Hausken, T., Little, T. J., Gentilcore, D., et al. (2009). Effects of the phases of the menstrual cycle on gastric emptying, glycemia, plasma GLP-1 and insulin, and energy intake in healthy lean women. Am. J. Physiol. Gastrointest. Liver Physiol. 297, G602-G610. doi: 10.1152/ajpgi. 00051.2009

Brooks, M., Kristensen, K., van Benthem, K., Magnusson, A., Berg, C. W., Nielsen, A., et al. (2017). glmmTMB balances speed and flexibility among packages for zero-inflated generalized linear mixed modeling. R. J. 9, 378-400. doi: 10.32614/ rj-2017-066

Brunstrom, J. M. (2014). Mind over platter: pre-meal planning and the control of meal size in humans. Int. J. Obes. 38(Suppl. 1), S9-S12. doi: 10.1038/ijo.2014.83

Buffenstein, R., Poppitt, S. D., McDevitt, R. M., and Prentice, A. M. (1995). Food intake and the menstrual cycle: a retrospective analysis, with implications for appetite research. Physiol. Behav. 58, 1067-1077. doi: 10.1016/0031-9384(95) 02003-9

Burger, K. S., and Stice, E. (2011). Variability in reward responsivity and obesity: evidence from brain imaging studies. Curr. Drug Abuse Rev. 4, 182-189. doi: 10.2174/1874473711104030182

Carnell, S., Gibson, C., Benson, L., Ochner, C. N., and Geliebter, A. (2012). Neuroimaging and obesity: current knowledge and future directions. Obes. Rev. 13, 43-56. doi: 10.1111/j.1467-789X.2011.00927.x

Craig, C. L., Marshall, A. L., Sjöström, M., Bauman, A. E., Booth, M. L., Ainsworth, B. E., et al. (2013). International physical activity questionnaire: 12-country reliability and validity. Med. Sci. Sports Exerc. 35, 1381-1395.

Cribari-Neto, F., and Zeileis, A. (2010). Beta regression in R. J. Statist. Softw. 34, $1-24$.

de Araujo, I. E., Schatzker, M., and Small, D. M. (2020). Rethinking food reward. Annu. Rev. Psychol. 71, 139-164. doi: 10.1007/978-1-137-21747-9_8

Dreher, J.-C., Schmidt, P. J., Kohn, P., Furman, D., Rubinow, D., and Berman, K. F. (2007). Menstrual cycle phase modulates reward-related neural function in women. Proc. Natl. Acad. Sci. U.S.A. 104:2465. doi: 10.1073/pnas.0605569104

Duffey, K. J., and Popkin, B. M. (2011). Energy density, portion size, and eating occasions: contributions to increased energy intake in the United States, 19772006. PLoS Med. 8:e1001050. doi: 10.1371/journal.pmed.1001050

Dye, L., and Blundell, J. E. (1997). Menstrual cycle and appetite control: implications for weight regulation. Hum. Reprod. 12, 1142-1151. doi: 10.1093/ humrep/12.6.1142

Eertmans, A., Victoir, A., Vansant, G., and Van den Bergh, O. (2005). Food-related personality traits, food choice motives and food intake: mediator and moderator relationships. Food Qual. Prefer. 16, 714-726. doi: 10.1016/j.foodqual.2005.04. 007

Eglitis-media (2022). Average Height and Weight by Country. Available online at: https://www.worlddata.info/average-bodyheight.php (accessed January 15, 2022).

Fairburn, C. G., Cooper, Z., and O'Connor, M. (2014). Eating Disorder Examination, Edition 17.0D. Available online at: https://www.corc.uk.net/ media/1951/ede_170d.pdf (accessed August 04, 2020).

Fong, Y., Huang, Y., Lemos, M. P., and McElrath, M. J. (2018). Rank-based twosample tests for paired data with missing values. Biostatistics 19, 281-294. doi: 10.1093/biostatistics/kxx039

Frank, T. C., Kim, G. L., Krzemien, A., and Van Vugt, D. A. (2010). Effect of menstrual cycle phase on corticolimbic brain activation by visual food cues. Brain Res. 1363, 81-92. doi: 10.1016/j.brainres.2010.09.071

Frye, C. A., and Demolar, G. L. (1994). Menstrual cycle and sex differences influence salt preference. Physiol. Behav. 55, 193-197. doi: 10.1016/00319384(94)90031-0

Geiger, B. M., Haburcak, M., Avena, N. M., Moyer, M. C., Hoebel, B. G., and Pothos, E. N. (2009). Deficits of mesolimbic dopamine neurotransmission in rat dietary obesity. Neuroscience 159, 1193-1199. doi: 10.1016/j.neuroscience.2009.02.007
Gobbi, S., Weber, S. C., Graf, G., Hinz, D., Asarian, L., Geary, N., et al. (2020). Reduced neural satiety responses in women affected by obesity. Neuroscience 447, 94-112. doi: 10.1016/j.neuroscience.2020.07.022

Gorczyca, A. M., Sjaarda, L. A., Mitchell, E. M., Perkins, N. J., Schliep, K. C., Wactawski-Wende, J., et al. (2016). Changes in macronutrient, micronutrient, and food group intakes throughout the menstrual cycle in healthy, premenopausal women. Eur. J. Nutr. 55, 1181-1188. doi: 10.1007/ s00394-015-0931-0

Hardikar, S., Höchenberger, R., Villringer, A., and Ohla, K. (2017). Higher sensitivity to sweet and salty taste in obese compared to lean individuals. Appetite 111, 158-165. doi: 10.1016/j.appet.2016.12.017

Kassambara, A. (2020). rstatix: Pipe-Friendly Framework for Basic Statistical Tests. $R$ Package Version 0.6.0. Available online at: https://rpkgs.datanovia.com/ rstatix/ (accessed March 04, 2021).

Kleinridders, A., and Pothos, E. N. (2019). Impact of brain insulin signaling on dopamine function, food intake, reward, and emotional behavior. Curr. Nutr. Rep. 8, 83-91. doi: 10.1007/s13668-019-0276-z

Klump, K. L., Keel, P. K., Racine, S. E., Burt, S. A., Neale, M., Sisk, C. L., et al. (2013). The interactive effects of estrogen and progesterone on changes in emotional eating across the menstrual cycle. J. Abnorm. Psychol. 122, 131-137. doi: $10.1037 / \mathrm{a} 0029524$

List, J. A., and Shogren, J. F. (1999). Price information and bidding behavior in repeated second-price auctions. Am. J. Agric. Econ. 81, 942-949. doi: 10.2307/ 1244336

Lowe, C. J., Kolev, D., and Hall, P. A. (2016). An exploration of exercise-induced cognitive enhancement and transfer effects to dietary self-control. Brain Cogn. 110, 102-111. doi: 10.1016/j.bandc.2016.04.008

Lüdecke, D. (2018). ggeffects: tidy data frames of marginal effects from regression models. J. Open Source Softw. 3:772.

Martini, M. C., Lampe, J. W., Slavin, J. L., and Kurzer, M. S. (1994). Effect of the menstrual cycle on energy and nutrient intake. Am. J. Clin. Nutr. 60, 895-899. doi: $10.1093 /$ ajcn/60.6.895

Mattes, R. D. (2018). Snacking: a cause for concern. Physiol. Behav. 193(Pt B), 279-283. doi: 10.1016/j.physbeh.2018.02.010

Miller, E. K., and Cohen, J. D. (2001). An integrative theory of prefrontal cortex function. Annu. Rev. Neurosci. 24, 167-202.

Motoki, K., and Suzuki, S. (2020). Extrinsic factors underlying food valuation in the human brain. Front. Behav. Neurosci. 14:131. doi: 10.3389/fnbeh.2020.00131

NCD Risk Factor Collaboration (2016). Trends in adult body-mass index in 200 countries from 1975 to 2014: a pooled analysis of 1698 population-based measurement studies with 19.2 million participants. Lancet 387, 1377-1396. doi: 10.1016/S0140-6736(16)30054-X

Niv, Y., Joel, D., and Dayan, P. (2006). A normative perspective on motivation. Trends Cogn. Sci. 10, 375-381. doi: 10.1016/j.tics.2006.06.010

Parraga, I. M. (1990). Determinants of food consumption. J. Am. Diet. Assoc. 90, 661-663.

Plassmann, H., O’Doherty, J., and Rangel, A. (2007). Orbitofrontal cortex encodes willingness to pay in everyday economic transactions. J. Neurosci. 27, 99849988. doi: 10.1523/JNEUROSCI.2131-07.2007

R Core Team (2013). R: A Language and Environment for Statistical Computing. Vienna: R Foundation for Statistical Computing.

Rangel, A., Camerer, C., and Montague, P. R. (2008). A framework for studying the neurobiology of value-based decision making. Nat. Rev. Neurosci. 9, 545-556. doi: $10.1038 / \mathrm{nrn} 2357$

Reichenberger, J., Richard, A., Smyth, J. M., Fischer, D., Pollatos, O., and Blechert, J. (2018). It's craving time: time of day effects on momentary hunger and food craving in daily life. Nutrition 55-56, 15-20. doi: 10.1016/j.nut.2018. 03.048

Richard, J. E., López-Ferreras, L., Anderberg, R. H., Olandersson, K., and Skibicka, K. P. (2017). Estradiol is a critical regulator of food-reward behavior. Psychoneuroendocrinology 78, 193-202. doi: 10.1016/j.psyneuen.2017.01.014

Rosenthal, R. (1994). "Parametric measures of effect size," in The Handbook of Research Synthesis, eds H. Cooper and L. V. Hedges (New York, NY: Russell Sage Foundation), 237.

Smithson, M., and Verkuilen, J. (2006). A better lemon squeezer? Maximumlikelihood regression with beta-distributed dependent variables. Psychol. Methods 11, 54-71. doi: 10.1037/1082-989X.11.1.54 
Suzuki, S., Cross, L., and O'Doherty, J. P. (2017). Elucidating the underlying components of food valuation in the human orbitofrontal cortex. Nat. Neurosci. 20, 1780-1786. doi: 10.1038/s41593-017-0008-x

Tang, D. W., Fellows, L. K., and Dagher, A. (2014). Behavioral and neural valuation of foods is driven by implicit knowledge of caloric content. Psychol. Sci. 25, 2168-2176. doi: 10.1177/0956797614552081

The World Health Organization [WHO] (2022). Body Mass Index (BMI). Geneva: World Health Organization.

Wang, G.-J., Volkow, N. D., Logan, J., Pappas, N. R., Wong, C. T., Zhu, W., et al. (2001). Brain dopamine and obesity. Lancet 357, 354-357. doi: 10.1016/s01406736(00)03643-6

Wei, T., Simko, V., Levy, M., Xie, Y., Jin, Y., and Zemla, J. (2017). Package 'corrplot'. Statistician 56:e24.

Wickham, H. (ed.) (2006). ggplot.: An Implementation of the Grammar of Graphics in R. R Package Version 3.3.3.

Wills, T. A., Isasi, C. R., Mendoza, D., and Ainette, M. G. (2007). Self-control constructs related to measures of dietary intake and physical activity in adolescents. J. Adolesc. Health 41, 551-558. doi: 10.1016/j.jadohealth.2007.06. 013

Yoest, K. E., Cummings, J. A., and Becker, J. B. (2014). Estradiol, dopamine and motivation. Cent. Nerv. Syst. Agents Med. Chem. 14, 83-89. doi: 10.2174/ 1871524914666141226103135
Yoest, K. E., Quigley, J. A., and Becker, J. B. (2018). Rapid effects of ovarian hormones in dorsal striatum and nucleus accumbens. Horm. Behav. 104, 119-129. doi: 10.1016/j.yhbeh.2018. 04.002

Conflict of Interest: The authors declare that the research was conducted in the absence of any commercial or financial relationships that could be construed as a potential conflict of interest.

Publisher's Note: All claims expressed in this article are solely those of the authors and do not necessarily represent those of their affiliated organizations, or those of the publisher, the editors and the reviewers. Any product that may be evaluated in this article, or claim that may be made by its manufacturer, is not guaranteed or endorsed by the publisher.

Copyright (c) 2022 Heuberger, Gobbi, Weber, Graf, Tobler, Asarian, Geary, Roth and Leeners. This is an open-access article distributed under the terms of the Creative Commons Attribution License (CC BY). The use, distribution or reproduction in other forums is permitted, provided the original author(s) and the copyright owner(s) are credited and that the original publication in this journal is cited, in accordance with accepted academic practice. No use, distribution or reproduction is permitted which does not comply with these terms. 